\title{
Planilha de ensino-aprendizagem para a determinação do fluxo de calor em um telhado com a inclusão dos efeitos da radiação na superfície externa através do método numérico da bisseção
}

\section{Teaching-learning sheet for determination of roof heat flow including the effects of radiation on the external surface using the bisection numerical method}

\section{Enseñanza-aprendizaje hoja de cálculo para la determinación de flujo de} calor en un techo con la inclusión de efectos de la radiación en la superficie externa utilizando el método numérico de bisección

Tiago dos Santos Gonçalves (tiago.santos@ifc.edu.br)

Universidade Regional Integrada do Alto Uruguai e das Missões (URI) IFC - Instituto Federal Catarinense

Carolina Elisa Demaman Oro (carolinae.oro@hotmail.com)

Universidade Regional Integrada do Alto Uruguai e das Missões (URI)

Rogério Marcos Dallago (dallago@uricer.edu.br)

Universidade Regional Integrada do Alto Uruguai e das Missões (URI)

\section{Luciana Dornelles Venquiaturo (venquiaruto@uricer.edu.br)}

Universidade Regional Integrada do Alto Uruguai e das Missões (URI)

Resumo: O uso de planilhas de ensino-aprendizagem no ensino presencial ou remoto fornece ao aluno uma nova perspectiva de ensino, pois auxilia no desenvolvimento da capacidade de avaliar as possibilidades e variáveis do estudo em diferentes situações, uma vez que a planilha é de fácil utilização e muito versátil. O objetivo do presente estudo foi demostrar de maneira simplificada como montar uma planilha utilizando os softwares Microsoft Excel ou LibreOffice Calc para a determinação do fluxo de calor em placa plana (telhado) com os efeitos da radiação na superfície externa utilizando o método numérico da Bisseção. O exercício proposto engloba diferentes disciplinas dos cursos de Engenharia e a planilha se torna uma aliada positiva para a explicação e compreensão dos assuntos abordados. Foram mostrados todos os passos que devem ser seguidos para a montagem e realização do exercício e foi obtido um fluxo de calor de $29,4335 \mathrm{~W} / \mathrm{m}^{2}$ nas condições propostas e 71 iterações foram necessárias.

Palavras-chave: Planilha eletrônica; Ensino-aprendizagem; Transferência de calor.

Abstract: The use of teaching-learning worksheets in face-to-face or remote teaching provides the student a new perspective. It assists in the development of the ability to

Recebido em: $17 / 03 / 2021$

Aceite em: 13/07/2021 
evaluate the possibilities and variables of the study in different situations, since the spreadsheet is easy to use and very versatile. The objective of the present study was to demonstrate in a simplified way how to assemble a spreadsheet using Microsoft Excel or LibreOffice Calc software for determining the heat flow in a roof with the effects of radiation on the external surface using the bisection numerical method. The proposed exercise encompasses disciplines from the Engineering courses and the spreadsheet becomes a positive ally for the explanation and understanding of the subjects covered. All the steps that must be followed to set up and perform the exercise were shown and a heat flow of $29.4335 \mathrm{~W} / \mathrm{m}^{2}$ was obtained under the proposed conditions with 71 iterations.

Keywords: Spreadsheet; Teaching-learning; Heat transfer.

Resumen: El uso de hojas de trabajo de enseñanza-aprendizaje en la enseñanza presencial o remota proporciona al estudiante una nueva perspectiva docente, ya que ayuda a desarrollar la capacidad de evaluar las posibilidades y variables del estudio en diferentes situaciones, desde la hoja de trabajo. es fácil de usar y muy versátil. El objetivo del presente estudio fue demostrar de manera simplificada cómo ensamblar una hoja de cálculo utilizando el software Microsoft Excel o LibreOffice Calc para determinar el flujo de calor en una placa plana (techo) con los efectos de la radiación en la superficie externa mediante el método numérico. de bisección. El ejercicio propuesto engloba diferentes disciplinas de los cursos de Ingeniería y la hoja de trabajo se convierte en un aliado positivo para la explicación y comprensión de los temas tratados. Se mostraron todos los pasos que se deben seguir para montar y realizar el ejercicio y se obtuvo un flujo de calor de $29,4335 \mathrm{~W} / \mathrm{m}^{2}$ en las condiciones propuestas y fueron necesarias 71 iteraciones.

Palabras-clave: hoja electrónica; Enseñanza-aprendizaje; Transferencia de calor.

\section{INTRODUÇÃO}

A transmissão de calor é um fenômeno bastante rotineiro, aparecendo de várias formas em nosso dia a dia. A transferência de calor consiste na troca entre a energia térmica de dois corpos, sendo do corpo mais quente para o mais frio. Existem três formas de transmissão de calor, sendo por condução, convecção ou radiação (INCROPERA et al., 2013; RABELER; FEYISSA, 2019).

A condução pode ser definida como o processo pelo qual a energia é transferida de uma região de alta temperatura para uma região de temperatura mais baixa dentro de um meio (sólido, líquido ou gás) ou entre diferentes meios de em contato direto. A convecção pode ser definida como o processo pelo qual a energia é transferida de porções quentes para porções frias de um fluido, ou entre uma superfície e um fluido, por meio da ação combinada de condução de calor, armazenamento de energia e movimento. Já a radiação pode ser definida como o processo pelo qual o calor é transferido de uma superfície em

Recebido em: 17/03/2021

Aceite em: 13/07/2021 
alta temperatura para uma superfície em temperatura mais baixa quando tais superfícies são separadas no espaço, mesmo que haja um vácuo entre elas. A energia assim transferida é chamada de radiação térmica e é feita na forma de ondas eletromagnéticas (INCROPERA et al., 2013; KHAN et al., 2020; NAKAGAWA; OCHIAI, 2015; SIGUEMOTO et al., 2018).

O entendimento da natureza de cada forma de transferência de calor é de suma importância para fins de projeto. Negligenciar alguma forma de transmissão, desde que esta negligência não ocorra em uma etapa ou resistência relevante, pode limitar o processo e o entendimento de todas as variáveis envolvidas no mesmo.

Em um telhado ou em uma parede de construção ocorre a transferência de calor sob as três maneiras simultaneamente. Contudo, para incluir o processo de radiação na metodologia de estado estacionário, com o objetivo de projetar sistemas de refrigeração, são necessárias considerações no processo de radiação para que se possa estimar o fluxo de calor, assim como, o perfil de temperatura da parede, que nem sempre podem estar conforme a realidade dos fatos. Isso pode ocasionar erros que podem inviabilizar o projeto, tanto do ponto de vista econômico quanto de manter o sistema em conformidade perante os parâmetros estipulados.

Como ferramenta de ensino e aprendizagem será abordada a utilização de equações que relacionam resistências térmicas por condução, convecção e radiação, sob a análise de resistência equivalente em série e em paralelo, com a finalidade de avaliar as influências de cada resistência para o fluxo de troca de calor e perfil de temperatura deste processo no telhado.

A utilização de planilha eletrônica como ferramenta de ensino-aprendizagem nos remete a métodos numéricos, ampliando assim o horizonte de aprendizagem e complementando esta atividade de importância para as engenharias em geral. A transferência de calor em paredes serve para simular câmaras frias, estocagem e até mesmo os sistemas de trocas de calor em aviários.

O uso do computador no ensino conduz o aluno a um universo de informações que possibilita desenvolver a imaginação, criatividade, percepção, raciocínio e as competências necessárias para a produção e transmissão de conhecimentos (ARAÚJO et al., 2005). Assim, quando o estudante pode construir o seu conhecimento desenvolvendo Recebido em: 17/03/2021

Aceite em: 13/07/2021 
atividades, e essas atividades desafiam os alunos a comparar as suas previsões, têm-se a Metodologia Ativa (ROCHA; MARANGHELLO; LUCCHESE, 2014). Contudo, para que esse objetivo seja alcançado, é necessário haver mudanças dos professores em relação às suas metodologias de ensino (SUÁREZ SILVA; FORTES BRAIDANTE, 2018). Outro ponto interessante para ser levado em consideração é o de que quando um professor passa a inovar e obter resultados positivos com o auxílio de tecnologias e planilhas de ensino-aprendizagem, ele se torna referência para que outros professores reflitam sobre as suas práticas docentes, com o intuito de aprimorar suas aulas e abordagens pelo uso das diversas tecnologias disponíveis. Ou seja, o uso de planilhas de ensino-aprendizagem influencia tanto os alunos quanto os professores positivamente.

Para os estudantes que pretendem ingressar na área empresarial, sugere-se o aprendizado de planilhas eletrônicas, pois as empresas têm obtido vantagens competitivas com o uso das mesmas (SHITSUKA et al., 2008). Dessa forma, a universidade deve ser um local que propicie o estudo da aplicabilidade dos assunto abordados em sala de aula (MORO; COPPI; PRSYBYCIEM, 2019). As planilhas de ensino-aprendizagem podem ser utilizadas tanto em aulas presenciais quanto em aulas remotas e fazem com que o aluno participe de forma ativa no processo que envolve a aquisição de conhecimentos. $\mathrm{O}$ uso das tecnologias de forma remota devido a pandemia ocasionada pela COVID-19 no ano de 2020 aumentou consideravelmente. Segundo Pereira e de Barros (2020), todo o cenário ocasionado pelo novo coronavírus trouxe uma oportunidade pedagógica. Cabe aos educadores comprometidos na produção de materiais didáticos de qualidade integrarem e interpretarem esse processo para não ficarem para trás. Contudo, poucas instituições propuseram iniciativas inovadoras para o ensino de seus alunos. Como relatado por Figueira e Veit (2004), o uso de planilhas eletrônicas se deve aos seguintes fatos: são altamente ajustáveis às necessidades em vários campos de atividade; permitem cálculos numéricos sem conhecimento de qualquer linguagem de programação ou metáfora simbólica; cálculos com planilhas costumam ser inteligíveis e apreciados por professores, além de propiciarem a construção de gráficos (FIGUEIRA; VEIT, 2004).

Alguns alunos podem observar uma situação crítica em relação às aulas remotas no período de pandemia, o que pode provocar desestímulo e falta de perspectiva em relação às suas vidas e ao futuro (PEREIRA; DE BARROS, 2020). Nessa perspectiva, as 
instituições de ensino passaram a utilizar alternativas de ensino, como o ensino remoto pelo uso de ferramentas digitais (SANTOS et al., 2020). No presente estudo, foi possível observar que os alunos de graduação muitas vezes não dominam de forma suficiente a teoria dos conteúdos e das planilhas para compreensão e elaboração dos exercícios propostos em aula na forma de planilhas de estudo. Neste contexto é apresentado o passo a passo para a resolução do exercício proposto, bem como os resultados obtidos utilizando a planilha de ensino-aprendizagem, a qual pode ser facilmente usada em aulas presenciais e remotas.

Dessa forma, o objetivo do presente estudo é abordar a metodologia de avaliação de transferência de calor em uma parede plana através de método numérico da bisseção utilizando uma planilha eletrônica como ferramenta de ensino-aprendizagem. Será determinado o fluxo de calor que atravessa um telhado com a inclusão dos efeitos da radiação na superfície externa do mesmo. Essa planilha envolve os conteúdos referentes às disciplinas de cálculo numérico, termodinâmica e transferência de calor de forma simples para posterior uso em sala de aula.

\section{METODOLOGIA}

O passo a passo da elaboração da planilha eletrônica é demostrado a seguir. Ressalta-se que a aplicação desta ferramenta (elaboração e aplicação) foi avaliada com os bolsistas de iniciação científica de uma turma de Engenharia Química, cursando o $9^{\circ}$ semestre, a qual na sequencia iria para o estágio obrigatório na indústria, no ano de 2020. A elaboração da planilha foi realizada com os alunos de forma online, mediante da qual eles acompanharam o professor montar a planilha e simultaneamente montaram as suas planilhas com os dados do exercício proposto. Como aplicação e discussão da ferramenta, outros valores de entrada foram testados afim de mostrar a facilidade de implementação da planilha em diferentes situações. Alguns alunos deram depoimento de suas experiências após a presente aula e, com a permissão dos mesmos, os depoimentos estão no final do presente estudo.

\subsection{Cálculo de raiz da temperatura externa de um telhado em que a radiação não} pode ser desprezada

Recebido em: $17 / 03 / 2021$

Aceite em: 13/07/2021 
O telhado e suas resistências respectivas está representado através da Figura 1. O telhado apresenta associação em série e em paralelo (convecção externa e radiação). Contudo, a informação da temperatura externa do metal que está diretamente associado ao processo de radiação não é conhecida. Para encontrar essa informação, é preciso utilizar um método numérico em que a temperatura externa do telhado é uma entrada para o fluxo de calor. Assim, com posse do dado de fluxo de calor e das temperaturas interna e externa (ar ambiente) do telhado, é possível realizar a iteração necessária com o objetivo de avaliar se o gradiente de temperatura é atingido.

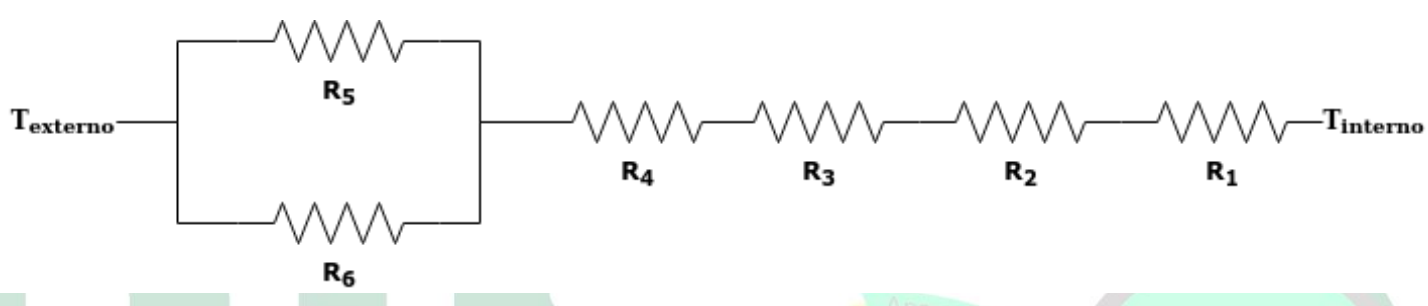

Figura 1 - Representação da associação de resistências térmicas do telhado. Fonte: Os autores (2021).

Neste processo de troca térmica há seis resistências denominadas de $\mathrm{R}_{1}, \mathrm{R}_{2}, \mathrm{R}_{3}, \mathrm{R}_{4}$, $\mathrm{R}_{5}$ e $\mathrm{R}_{6}$, com as seguintes descrições:

- $\quad \mathrm{R}_{1}$ é a resistência da convecção interna do telhado, calculada conforme Equação (1).

$$
R_{1}=\frac{1}{h_{i}}
$$

Onde: $R_{1}$ é a resistência ao fluxo de calor da convecção interna $\left({ }^{\circ} \mathrm{C} \mathrm{m} / \mathrm{W}\right)$ e $h_{i}{ }^{\prime} \mathrm{r}$ o coeficiente de troca térmica por convecção na parte interna do telhado $\left(\mathrm{W} / \mathrm{m}^{2}{ }^{\circ} \mathrm{C}\right)$.

- $\quad \mathrm{R}_{2}$ é a resistência da condução do forro (neste exemplo, o forro é de policloreto de vinila, PVC) da construção do telhado, calculada conforme Equação (2).

$$
R_{2}=\frac{L_{P V C}}{k_{P V C}}
$$


Onde: $R_{2}$ é a resistência ao fluxo de calor da condução do PVC $\left({ }^{\circ} \mathrm{C} \mathrm{m} / \mathrm{W}\right), L_{P V C}$ é a espessura da placa de PVC $(\mathrm{m})$, e $k_{P V C}$ é a condutividade térmica da placa de PVC $(\mathrm{W} / \mathrm{m}$ $\left.{ }^{\circ} \mathrm{C}\right)$.

- $\quad \mathrm{R}_{3}$ é a resistência da condução do isolamento térmico da construção do telhado, calculada conforme Equação (3).

$$
R_{3}=\frac{L_{I S O L A M E N T O}}{k_{\text {ISOLAMENTO }}}
$$

Onde: $R_{3}$ é a resistência ao fluxo de calor da condução do isolamento $\left({ }^{\circ} \mathrm{C} \mathrm{m}^{2} / \mathrm{W}\right)$, $L_{\text {ISOLAMENTO }}$ é a espessura da placa do isolamento (m), e $k_{\text {ISOLAMENTO }}$ é a condutividade térmica da placa do isolamento $\left(\mathrm{W} / \mathrm{m}{ }^{\circ} \mathrm{C}\right)$.

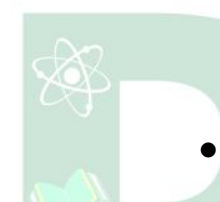

$\mathrm{R}_{4}$ é a resistência da condução do metal da construção do telhado, calculada conforme Equação (4).

$$
R_{4}=\frac{L_{M E T A L}}{k_{M E T A L}}
$$

Onde: $R_{4}$ é a resistência ao fluxo de calor da condução do isolamento $\left({ }^{\circ} \mathrm{C} \mathrm{m}{ }^{2} / \mathrm{W}\right), L_{M E T A L}$ é a espessura da placa do metal (m), e $k_{M E T A L}$ é a condutividade térmica da placa do metal $\left(\mathrm{W} / \mathrm{m}^{\circ} \mathrm{C}\right)$.

- $\quad \mathrm{R}_{5}$ é a resistência da convecção externa do telhado, calculada conforme Equação (5).

$$
R_{5}=\frac{1}{h_{e}}
$$

Onde: $R_{5}$ é a resistência ao fluxo de calor da convecção externa $\left({ }^{\circ} \mathrm{C} \mathrm{m} / \mathrm{W}\right)$ e $h_{e}$ é o coeficiente de troca térmica por convecção na parte externa do telhado $\left(\mathrm{W} / \mathrm{m}^{2}{ }^{\circ} \mathrm{C}\right)$.

- $\quad \mathrm{R}_{6}$ é a resistência da radiação externa do telhado, calculada conforme Equação (6). 


$$
R_{6}=\frac{1}{h_{R A D}}
$$

Onde: $R_{6}$ é a resistência ao fluxo de calor da radiação externa $\left({ }^{\circ} \mathrm{C} \mathrm{m} / \mathrm{W}\right)$ e $h_{R A D}$ é o coeficiente de troca térmica por radiação na parte externa do telhado $\left(\mathrm{W} / \mathrm{m}^{2}{ }^{\circ} \mathrm{C}\right)$, sendo calculada conforme Equação 7.

A Equação (7) é uma função da temperatura externa do telhado, denominada de $\mathrm{T}_{2}$, e da temperatura do ar atmosférico que está em contato com o telhado, denominada de $\mathrm{T}_{1}$.

$$
h_{R A D}=\frac{\sigma \varepsilon\left(T_{1}^{4}-T_{2}^{4}\right)}{\left(T_{1}-T_{2}\right)}
$$

Onde: $h_{R A D}$ é o coeficiente de troca térmica por radiação na parte externa do telhado $\left(\mathrm{W} / \mathrm{m}^{2}{ }^{\circ} \mathrm{C}\right), \sigma$ é a constante de Stefan-Boltzmann $\left(5,67 \times 10^{-8} \mathrm{~W} / \mathrm{m}^{2} \mathrm{~K}^{4}\right)$ - homenagem a Stefan que descobriu esta relação experimentalmente em 1879 e Boltzmann que realizou a dedução teórica sobre este fenômeno em 1884, $\varepsilon$ é a emissividade do corpo negro (sem unidade) - a emissividade varia de 0 para corpo branco a 1 para corpo negro, $T_{1}$ é a temperatura na escala absoluta (Kelvin) do meio ambiente, ou seja $\mathrm{T}($ Kelvin $)=\mathrm{T}\left({ }^{\circ} \mathrm{C}\right)$ $+273,15$, e $T_{2}$ é a temperatura na escala absoluta (Kelvin) da parte externa do telhado, ou seja $\mathrm{T}($ Kelvin $)=\mathrm{T}\left({ }^{\circ} \mathrm{C}\right)+273,15$.

As resistências $\mathrm{R}_{5}$ e $\mathrm{R}_{6}$ estão em paralelo, portanto é preciso calcular a resistência equivalente $\left(R_{E Q}\right)$ que produz o mesmo efeito destas duas resistências sobre o fluxo de calor que atravessa o telhado, como descrito através da Equação (8).

$$
\frac{1}{R_{E Q}}=\sum_{i=1}^{i=N}\left(\frac{1}{R_{i}}\right)=\frac{1}{R_{5}}+\frac{1}{R_{6}}
$$

Onde: $R_{E Q}$ é a resistência equivalente $\left({ }^{\circ} \mathrm{C} \mathrm{m}^{2} / \mathrm{W}\right)$ para o fluxo de calor em paralelo da convecção externa e da radiação externa, $R_{5}$ é a resistência da convecção externa $\left({ }^{\circ} \mathrm{C}\right.$ $\left.\mathrm{m}^{2} / \mathrm{W}\right)$, conforme Equação 5 , e $R_{6}$ é a resistência da radiação externa $\left({ }^{\circ} \mathrm{C} \mathrm{m}^{2} / \mathrm{W}\right)$, conforme Equação (6).

Recebido em: 17/03/2021

Aceite em: 13/07/2021 
Assim o fluxo de calor $\left(\mathrm{W} / \mathrm{m}^{2}\right)$ que atravessa o telhado será dado pela Equação (9), como representado a seguir.

$$
q=\frac{T_{1}-T_{2}}{R_{E Q}}
$$

Onde: $q$ é o fluxo de calor $\left(\mathrm{W} / \mathrm{m}^{2}\right)$ que atravessa o telhado, $T_{1}$ é a temperatura do meio ambiente $\left({ }^{\circ} \mathrm{C}\right), T_{2}$ é a temperatura externa do telhado $\left({ }^{\circ} \mathrm{C}\right)$, e $R_{E Q}$ é a resistência equivalente $\left({ }^{\circ} \mathrm{C} \mathrm{m} / \mathrm{W}\right)$ para o fluxo de calor.

As resistências $\left(R_{1}, R_{2}, R_{3}, R_{4}\right.$ e $\left.R_{E Q}\right)$ estão em série. Dessa forma, a resistência total $\left(R_{\text {TOTAL }}\right)$ é calculada conforme representado pela Equação (10).

$$
R_{\text {TOTAL }}=\sum_{i=1}^{i=N}\left(R_{i}\right)=R_{1}+R_{2}+R_{3}+R_{4}+R_{E Q}
$$

Onde: $R_{1}$ é a resistência da convecção interna $\left({ }^{\circ} \mathrm{C} \mathrm{m}^{2} / \mathrm{W}\right)$, conforme Equação $1, R_{2}$ é a resistência da condução do $\mathrm{PVC}\left({ }^{\circ} \mathrm{C} \mathrm{m}^{2} / \mathrm{W}\right)$, conforme Equação $2, R_{3}$ é a resistência da condução do Isolamento $\left({ }^{\circ} \mathrm{C} \mathrm{m}^{2} / \mathrm{W}\right.$ ), conforme Equação $3, R_{4}$ é a resistência da condução do metal $\left({ }^{\circ} \mathrm{C} \mathrm{m} / \mathrm{W}\right)$, conforme Equação 4 , e $R_{E Q}$ é a resistência equivalente $\left({ }^{\circ} \mathrm{C} \mathrm{m} / \mathrm{W}\right)$ para o fluxo de calor em paralelo da convecção externa e da radiação externa, conforme Equação (8).

O modelo de resistência equivalente em série ocorre quando há uma sequência de resistências no sistema ao longo do gradiente de temperatura, muito utilizado em processos de construção de paredes e de isolamentos.

O modelo de resistência equivalente em paralelo ocorre quando há dois processos simultâneos de troca térmica em uma mesma região do gradiente de temperatura, muito utilizado quando ocorre processos de convecção e radiação ao mesmo tempo, seja internamente em caldeiras ou externamente para linhas de refrigeração entre compressores e condensadores.

Estas equações podem ser utilizadas em qualquer geometria disponível para resolução analítica ou numérica de transporte de calor. Lembrando que a resistência Recebido em: 17/03/2021

Aceite em: 13/07/2021 
equivalente em série é sempre maior do que todas as resistências e a resistência equivalente em paralelo é sempre menor do que todas as resistências.

Como o fluxo de calor que atravessa todas as resistências do telhado possui o mesmo valor, por ser tratar de um sistema em série, o gradiente de temperatura total do sistema é gerado através do produto entre o fluxo de calor (Equação 9) e a Resistência Total do sistema (Equação 10), conforme observado pela Equação (11).

$$
\Delta T_{\text {TOTAL }}=T_{\text {externo }}-T_{\text {interno }}=q \times R_{\text {TOTAL }}
$$

Onde: $T_{\text {externo }}$ é a temperatura do meio ambiente externo ao telhado $\left({ }^{\circ} \mathrm{C}\right), T_{\text {interno }}$ é a temperatura do meio ambiente interno ao telhado $\left({ }^{\circ} \mathrm{C}\right), q$ é o fluxo de calor $\left(\mathrm{W} / \mathrm{m}^{2}\right)$, conforme Equação 9, e $R_{\text {TотAL }}$ é a resistência total do sistema $\left({ }^{\circ} \mathrm{C} \mathrm{m} / \mathrm{W}\right)$, conforme Equação (10).

A equação que devemos encontrar a raiz (fluxo de calor) em função da temperatura externa do telhado $\left(T_{E X T}\right)$ é descrita através da Equação (12).

Com as informações de entrada do sistema: Temperatura interna, Temperatura externa, Coeficiente de convecção interna, Coeficiente de convecção externa, Espessura e condutividade térmica do PVC, Espessura e condutividade térmica do isolamento, Espessura e condutividade térmica do metal e emissividade do telhado, $T_{E X T}$ é a solução da Equação (12) em um processo iterativo cujo a metodologia é o da bisseção.

$$
0=T_{\text {externo }}-T_{\text {interno }}-q \times R_{\text {TOTAL }}
$$

Os dados de entrada para o cálculo do fluxo de calor em um telhado com a inclusão dos efeitos da radiação na superfície externa são apresentados na Tabela 1. Esses dados foram colocados a fim de exemplificar o processo de radiação. Todas as entradas da Tabela 1 podem ser alteradas conforme o desejado para o desenvolvimento do exercício (outro material de isolamento, outro metal, etc.).

Tabela 1 - Dados de entrada para o desenvolvimento do cálculo de fluxo de calor com radiação

\begin{tabular}{lc}
\multicolumn{2}{c}{ utilizando o método da bisseção. } \\
\hline Variável & Valor de entrada \\
\hline Convecção interna $\left(\mathrm{W} / \mathrm{m}^{2}{ }^{\circ} \mathrm{C}\right)$ & 33 \\
\hline
\end{tabular}

Recebido em: $17 / 03 / 2021$

Aceite em: 13/07/2021 


\begin{tabular}{lc}
\hline $\mathrm{L}_{\mathrm{PVC}}(\mathrm{mm})$ & 5 \\
$\mathrm{k}_{\mathrm{PVC}}\left(\mathrm{W} / \mathrm{m}{ }^{\circ} \mathrm{C}\right)$ & 0,4 \\
$\mathrm{~L}_{\text {Isolamento }}(\mathrm{mm})$ & 35 \\
$\mathrm{k}_{\text {Isolamento }}\left(\mathrm{W} / \mathrm{m}{ }^{\circ} \mathrm{C}\right)$ & 0,05 \\
$\mathrm{~L}_{\text {Metal }}(\mathrm{mm})$ & 3 \\
$\mathrm{k}_{\text {Metal }}\left(\mathrm{W} / \mathrm{m}{ }^{\circ} \mathrm{C}\right)$ & 190 \\
Convecção externa $\left(\mathrm{W} / \mathrm{m}^{2}{ }^{\circ} \mathrm{C}\right)$ & 20 \\
Radiação $\left(\varepsilon_{\text {TELHADO })}\right.$ & 0,8 \\
Temperatura interna $\left({ }^{\circ} \mathrm{C}\right)$ & 22 \\
\hline
\end{tabular}

Fonte: Os autores (2021).

\subsection{Método da Bisseção}

O Método da Bisseção é um método de busca de raízes que divide repetidamente um intervalo, e então, seleciona um subintervalo contendo a raiz para processamento adicional. Trata-se de um método simples e robusto, relativamente lento quando comparado a métodos com os Métodos de Newton ou das Secantes, por causa de sua convergência linear.

Para a solução da Equação (12) do fluxo de calor no telhado, há uma dificuldade de encontrar a derivada da mesma, portanto a simples divisão do intervalo de temperaturas interna e externa, que são dados do problema $\left(T_{I N T}\right.$ e $\left.T_{E X T}\right)$ é mais adequada para o encontro da raiz.

O número de iterações $(n)$ necessário para o encontro da raiz da Equação 12 é apresentado através da Equação (13), em que $\varepsilon_{0}$ é o intervalo das temperaturas ( $\mathrm{T}_{\mathrm{INT}}$, $\mathrm{T}_{\mathrm{EXT}}$ ) e $\varepsilon$ é a margem de erro ou tolerância para este processo de cálculo de raiz. Com um erro associado $\varepsilon$ igual a $1 \times 10^{-20}$, portanto o número mínimo de iterações é uma função de gradiente de temperatura interna e externa do telhado, conforme dado pela Equação (13).

$$
n=\frac{\log \left(\varepsilon_{0}\right)-\log (\varepsilon)}{\log (2)}
$$

Este método pode ser usado para encontrar as raízes de uma função contínua $\mathbf{f}:[\mathbf{a}, \mathbf{b}]$ $\rightarrow \mathbf{R}, \mathbf{y}=\mathbf{f}(\mathbf{x})$, tendo $\mathbf{f}(\mathbf{a})$ e $\mathbf{f}(\mathbf{b})$ sinais opostos, ou seja, $\mathbf{f}(\mathbf{a}) \mathbf{x} \mathbf{f}(\mathbf{b})<\mathbf{0}$. Nestas condições, o teorema do valor intermediário garante a existência de uma raiz no intervalo. O método consiste em dividir o intervalo no seu ponto médio $(\mathbf{c}=(\mathbf{a}+\mathbf{b}) / \mathbf{2})$, e então verificar em qual dos dois subintervalos garante-se a existência de uma raiz. Para tanto, basta verificar se $\mathbf{f}(\mathbf{a}) \mathbf{x} \mathbf{f}(\mathbf{c})<\mathbf{0}$. Caso afirmativo, existe pelo menos uma raiz no intervalo (a,c), caso 
contrário garante-se a existência de uma raiz no intervalo [c, b). O procedimento é, então, repetido para o subintervalo correspondente à raiz até que $\mathbf{c}$ aproxime a raiz com a precisão desejada.

Todos os cálculos podem realizados utilizando os softwares Microsoft Excel ou LibreOffice Calc, e os resultados obtidos são apresentados nos resultados a seguir.

\section{RESULTADOS E DISCUSSÕES}

Para fins de explicação didática para os alunos, as conversões de unidades podem ser realizadas conforme são resolvidas as equações, como mostrado na Figura 2 com os dados da Tabela 1. Ao mostrar a conversão de unidades em uma aba separada na planilha, os alunos prestam maior atenção e se lembram dessa etapa como uma das mais fundamentais. Assim, deve-se sempre cuidar para que a unidade das variáveis seja compatível com as equações a serem empregadas para a resolução do exercício proposto.

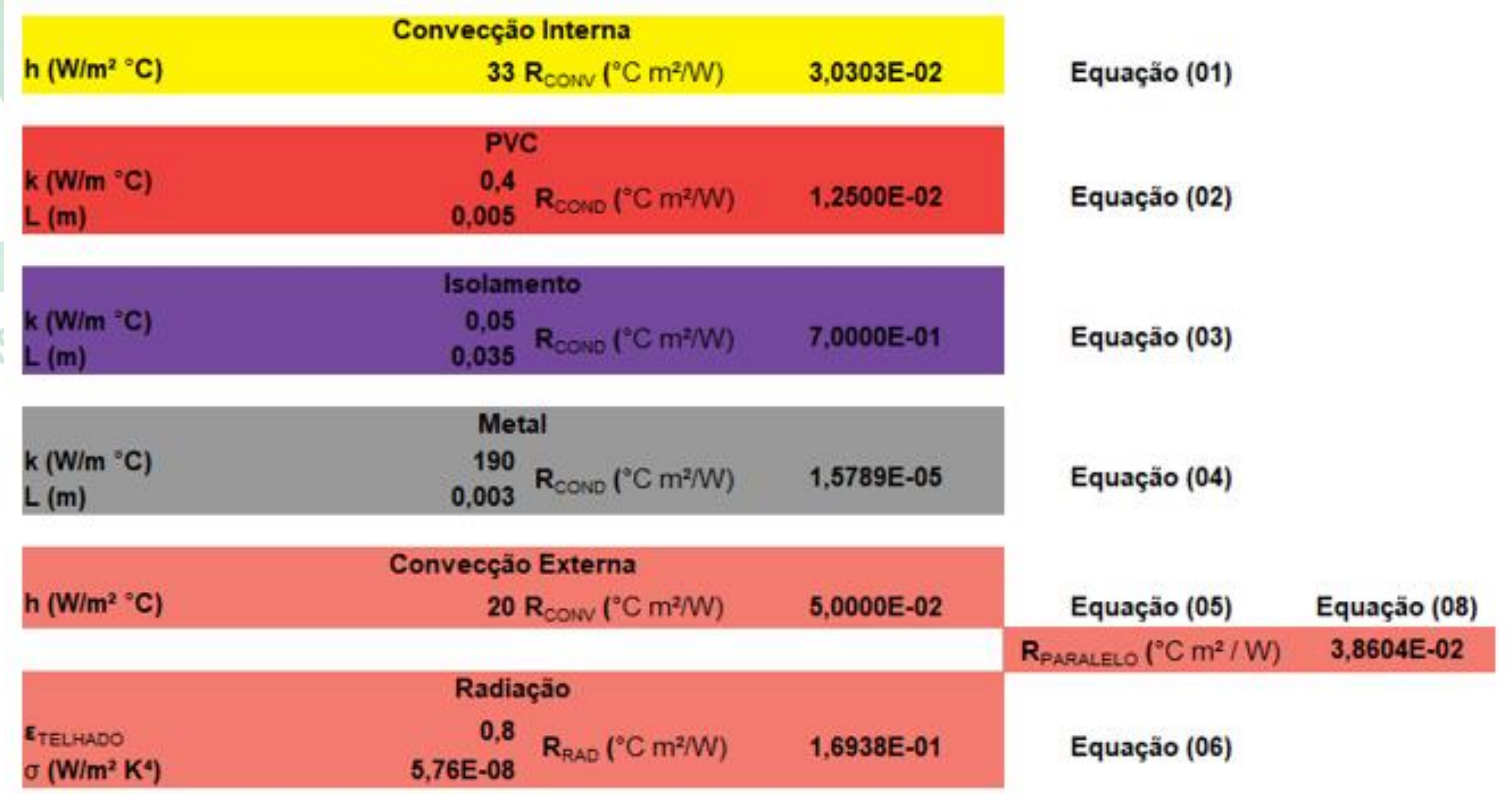

Fonte: Os autores (2021).

Figura 2 - Organização da planilha com as conversões de unidade para posterior cálculo das respectivas equações.

Através do uso da Equação 13 é possível calcular o intervalo, o erro e o número de iterações necessárias pelo método da Bisseção, como apresentado na Tabela 2. Para o presente estudo, foram necessárias 71 iterações (Figura 3) para encontrar a resposta do Recebido em: $17 / 03 / 2021$

Aceite em: 13/07/2021 
proposto problema com o menor erro possível. Com o uso da ferramenta proposta, problemas como esse apresentado se tornam fáceis de resolver e de conferir em sala de aula. Se o mesmo problema fosse resolvido no caderno, muito tempo seria gasto pelos alunos. Além disso, qualquer pequeno erro pode comprometer toda a resolução do exercício. Por isso, incentivar os alunos a utilizar ferramentas como o Microsoft Excel ou LibreOffice Calc, que está ao acesso da maioria, se torna necessário. Isso os incentiva a estudar projetos maiores e os ajuda no exercício do raciocínio lógico, uma vez que são várias etapas a serem levadas em consideração na resolução do presente exercício matemático que engloba conceitos de diferentes disciplinas.

Tabela 2 - Intervalo, erro e número de iterações necessárias para o desenvolvimento do presente estudo pelo método da Bisseção.

\begin{tabular}{lc}
\hline Respostas do método da Bisseção & Valores encontrados \\
\hline$\varepsilon_{0}$ (intervalo) $\left({ }^{\circ} \mathrm{C}\right)$ & 23 \\
$\varepsilon$ (erro) & $1,00 \mathrm{E}^{-20}$ \\
Número de Iterações & 71 \\
\hline
\end{tabular}

Fonte: Os autores (2021).

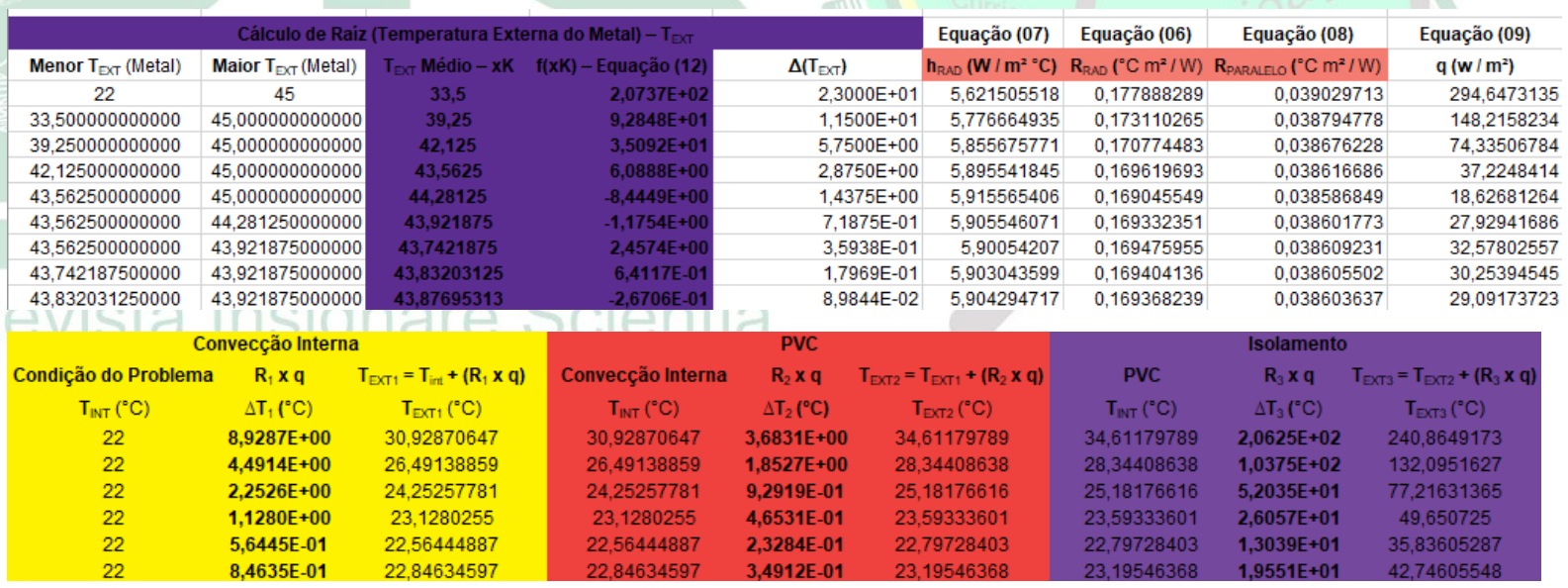

\begin{tabular}{|c|c|c|c|c|c|}
\hline \multicolumn{3}{|c|}{ Metal } & \multicolumn{3}{|c|}{ Radiação + Convecção Externa } \\
\hline Isolamento & $\mathrm{R}_{4} \times \mathrm{q}$ & $\mathrm{T}_{\mathrm{EXT} 4}=\mathrm{T}_{\mathrm{EXT} 3}+\left(\mathrm{R}_{4} \times q\right)$ & Metal & $\mathbf{R}_{\mathrm{EQ}} \times \mathbf{q}$ & $\mathrm{T}_{\mathrm{EXT5}}=\mathrm{T}_{\mathrm{EXT} 4}+\left(\mathbf{R}_{E \mathrm{EO}} \times \mathbf{q}\right)$ \\
\hline $\mathrm{T}_{\mathrm{INT}}\left({ }^{\circ} \mathrm{C}\right)$ & $\Delta \mathrm{T}_{4}\left({ }^{\circ} \mathrm{C}\right)$ & $\mathrm{T}_{\mathrm{DXT} 4}\left({ }^{\circ} \mathrm{C}\right)$ & $\mathrm{T}_{\mathrm{INT}}\left({ }^{\circ} \mathrm{C}\right)$ & $\Delta \mathrm{T}_{5}\left({ }^{\circ} \mathrm{C}\right)$ & $\mathrm{T}_{\mathrm{EXT5}}\left({ }^{\circ} \mathrm{C}\right)$ \\
\hline 240,86492 & $4,6523 E-03$ & 240,8695696 & 240,86957 & 11,5 & 252,3695696 \\
\hline 132,09516 & $2,3402 E-03$ & 132,097503 & 132,0975 & 5,75 & 137,847503 \\
\hline 77,216314 & 1,1737E-03 & 77,21748736 & 77,217487 & 2,875 & 80,09248736 \\
\hline 49,650725 & $5,8776 \mathrm{E}-04$ & 49,65131276 & 49,651313 & 1,4375 & 51,08881276 \\
\hline 35,836053 & $2,9411 \mathrm{E}-04$ & 35,83634698 & 35,836347 & 0,71875 & 36,55509698 \\
\hline
\end{tabular}

Fonte: Os autores (2021).

Figura 3 - Visão geral da tabela de ensino-aprendizagem montada com os alunos com todos os passos necessários e algumas das iterações pelo Método da Bisseção.

A Tabela 3 apresenta o perfil de temperatura em cada camada do telhado. É importante conhecer o perfil de temperatura, uma vez que é possível verificar a 
contribuição de cada camada na transferência de calor. A informação da temperatura externa do metal que está diretamente associado ao processo de radiação não foi fornecida. Por isso, a temperatura externa do metal do telhado é o ponto em que é necessário realizar a iteração, para que não seja feito hipóteses no processo.

Como resposta, foi encontrada uma temperatura externa de $45{ }^{\circ} \mathrm{C}$ (convecção externa + radiação). Com base nesse perfil de temperatura, os cálculos foram realizados e o fluxo de calor pode ser determinado.

Tabela 3 - Perfil de temperatura do telhado em cada camada do telhado em estudo.

\begin{tabular}{lcccc}
\hline \multicolumn{1}{c}{ Camada } & $\begin{array}{c}\mathbf{T}_{\text {Externa }} \\
\left({ }^{\circ} \mathbf{C}\right)\end{array}$ & $\begin{array}{c}\mathbf{R}\left({ }^{\circ} \mathbf{C}\right. \\
\left.\mathbf{m}^{2} / \mathbf{W}\right)\end{array}$ & $\Delta \mathbf{T}$ & $\begin{array}{c}\text { Tinterna } \\
\left({ }^{\circ} \mathbf{C}\right)\end{array}$ \\
\hline Convecção Externa + Radiação & 45,0000 & $3,8604 \mathrm{E}^{-02}$ & 1,1363 & 43,8637 \\
Metal & 43,8637 & $1,5789 \mathrm{E}^{-05}$ & 0,0005 & 43,8633 \\
Isolamento & 43,8633 & $7,0000 \mathrm{E}^{-01}$ & 20,603 & 23,2598 \\
& & & 4 & \\
PVC & 23,2598 & $1,2500 \mathrm{E}^{-02}$ & 0,3679 & 22,8919 \\
Convecção Interna & 22,8919 & $3,0303 \mathrm{E}^{-02}$ & 0,8919 & 22,0000 \\
\hline
\end{tabular}

Como resposta, foi obtido um fluxo de calor de $29,4335 \mathrm{~W} / \mathrm{m}^{2}$. Foi escolhido trabalhar com no mínimo 4 casas decimais para mostrar os resultados da realização desse exercício. Dessa forma, é fácil mostrar e explicar para os alunos sobre os erros que podem acontecer quando é realizado o mesmo exercício com arredondamento dos dados, como acontece ao realizar o exercício de forma manual.

A resolução de exercícios semelhantes de fluxo de calor em placa plana, uma vez que a tabela esteja montada no programa, pode ser realizada apenas trocando os valores iniciais da planilha. Isso retorna em tempo para o professor explicar em sala de aula todas as atribuições que os materiais possuem no fluxo de calor e sugerir desafios baseados no conteúdo proposto.

\section{Relato dos acadêmicos}

Ao término da disciplina os acadêmicos foram convidados a relatar sobre o aprendizado proporcionado pelo desenvolvimento e aplicação da planilha eletrônica Recebido em: $17 / 03 / 2021$

Aceite em: 13/07/2021 
construída empregando os softwares Microsoft Excel ou LibreOffice Calc. Solicitou-se ainda que os mesmos no decorrer do estágio obrigatório, relatassem a sua experiência com o uso desta tecnologia.

Grande parte dos acadêmicos relataram que o desenvolvimento e aplicação da planilha eletrônica proporcionou novos aprendizados envolvendo o uso de novas tecnologias baseadas no emprego de ferramentas acessíveis, normalmente disponíveis a todos os acadêmicos que dispõem de um computador. Em relação especifica a planilha apresentada no presente estudo, a mesma possibilitou verificar de forma rápida os efeitos de diferentes variáveis no processo de transferência de calor.

Indiretamente, a familiarização com os softwares e suas formas de programação se demonstrou um facilitador para o aprendizado e a aplicação em diversos conteúdos, conforme os relatos fornecidos durante e após o estágio curricular na Indústria:

Já usei um pouco de tudo que aprendi das aulas de Excel no meu estágio. Eu fiz os minigráficos em uma tabela de análise semanal o chefe gostou. Fiquei muito feliz (E1).

As aulas de Excel foram muito importantes pra a minha organização da faculdade e da minha vida pessoal. Muitas das matérias que tive após a prender a usar Excel acabaram ficando muito mais fáceis e simples apenas pelo fato de eu poder e saber utilizar essa ferramenta. As matérias de Termodinâmica aplicada, aplicações industriais do calor, calculo numérico entre diversas outras são exemplos disso. Além de ser muito importante para a minha bolsa de Iniciação cientifica (E2).

As aulas de Excel foram muito úteis nas disciplinas de termodinâmica e cálculo numérico, onde eram feitos cálculos de aproximação para encontrar raízes de funções a partir das repetições, a partir de métodos como o da bisseção, de Newton e das secantes. Também foi muito utilizado no estágio em indústria na avaliação de produtividade, onde todas as análises eram realizadas a partir do software Excel 2016. As aulas contribuíram muito na elaboração de fórmulas a partir de bancos de dados extensos e construção de gráficos para a estratificação dos desvios ocorridos (E3).

O conhecimento adquirido com o uso da presente planilha demonstrou ser de suma importância no que se refere a formação profissional, uma vez que o domínio dos softwares estudados aparece com frequência como requisito nas entrevistas de estágio, bem como em vagas de emprego na área das Engenharias.

\section{CONCLUSÕES}

Recebido em: $17 / 03 / 2021$

Aceite em: 13/07/2021 
A resolução de um exercício para determinar o fluxo de calor em um telhado com configuração de placa plana sem desprezar os efeitos da radiação foi realizado com sucesso através do emprego do Método da Bisseção em uma planilha de ensinoaprendizado, uma vez que todos os alunos conseguiram montar a sua planilha e resolver o exercício proposto. Foram mostrados todos os passos que devem ser seguidos para a montagem e realização do exercício. Essa planilha é uma ferramenta prática para explicar os conceitos da disciplina tanto de forma presencial como de forma remota, facilitando o aprendizado e minimizando o tempo de resolução do exercício com diferentes entradas pelos alunos, em comparação com a forma tradicional, isto é, a resolução de forma manual no caderno. Ou seja, com uma planilha base para um mesmo exercício, é possível, ao trocar o valor de qualquer uma das variáveis de entrada, já ter acesso à resposta automaticamente.

Além de todas as vantagens já mencionadas ao utilizar a planilha de ensinoaprendizagem em sala de aula, cabe destacar que a utilização da mesma gera o consequente aprendizado dos softwares para desenvolvimento da mesma. Essa habilidade estimula o aprendizado e uso das ferramentas para outras atividades e disciplinas, além de ser demandada pela indústria em processos seletivos de contratação. Dessa forma, o uso das planilhas de ensino também contribui para a formação do aluno para o mercado de trabalho.

\section{REFERÊNCIAS}

ARAÚJO, R. M. L. et al. A planilha excel como instrumento pedagógico na formação do professor de matemática. Zetetiké, v. 13, n. 23, p. 137-160, 2005.

FIGUEIRA, J. S.; VEIT, E. A. Usando o Excel para medidas de intervalo de tempo no laboratório de Física. Revista Brasileira de Ensino de Física, v. 26, n. 3, p. 203-211, 2004.

INCROPERA, F. P. et al. Principles of heat and mass transfer. New York, NY: John Wiley \& Sons, 2013.

KHAN, M. I. H. et al. Modelling of simultaneous heat and mass transfer considering the spatial distribution of air velocity during intermittent microwave convective drying.

International Journal of Heat and Mass Transfer, v. 153, p. 119668, 2020.

MORO, F. T.; COPPI, E. P.; PRSYBYCIEM, M. M. Construção de um biodigestor:

Recebido em: 17/03/2021

Aceite em: 13/07/2021 
uma proposta de ensino interdisciplinar para escolas do campo. Revista Insignare Scientia - RIS, v. 2, n. 1, p. 104-115, 2019.

NAKAGAWA, K.; OCHIAI, T. A mathematical model of multi-dimensional freezedrying for food products. Journal of Food Engineering, v. 161, p. 55-67, 2015.

PEREIRA, M. D.; DE BARROS, E. A. A educação e a escola em tempos de coronavírus. Scientia Vitae, v. 9, n. 28, p. 1-7, 2020.

RABELER, F.; FEYISSA, A. H. Modelling of food processes under uncertainty: Mechanistic 3D model of chicken meat roasting. Journal of Food Engineering, v. 262, p. 49-59, 2019.

ROCHA, F. S. DA; MARANGHELLO, G. F.; LUCCHESE, M. M. Acelerômetro eletrônico e a placa Arduino para ensino de física em tempo real. Caderno Brasileiro de Ensino de Física, v. 31, n. 1, p. 98-123, 2014.

SANTOS, E. T. DOS et al. Covid 19 E Os Impactos Na Educação: Percepções Sobre Brasil e Cuba. Hygeia - Revista Brasileira de Geografia Médica e da Saúde, Edição Especial COVID 19, p. 450-460, 2020.

SHITSUKA, R. et al. Uso de planilhas eletrônicas no ensino de tomada de decisão em cursos superiores. Exacta, v. 3, p. 125-131, 2008.

SIGUEMOTO, E. S. et al. Modeling of time-temperature history and enzymatic inactivation of cloudy apple juice in continuous flow microwave assisted pasteurization. Food and Bioproducts Processing, v. 111, p. 45-53, 2018.

SUÁREZ SILVA, J. A.; FORTES BRAIDANTE, M. E. Aprendizagem significativa: concepções na formação inicial de professores de Ciências. Revista Insignare Scientia - RIS, v. 1, n. 1, p. 1-22, 2018. 